\title{
Design of robust high-order superdirectivity for circular arrays with sensor gain and phase errors
}

\author{
Min Wang ${ }^{1 *}$ (D) Xiaochuan $\mathrm{Ma}^{2}$, Ping Yang ${ }^{1}$, Chengpeng Hao ${ }^{2}$, Xiujuan Feng ${ }^{1}$ and Yue Zhang ${ }^{1}$
}

\begin{abstract}
Though the high-order superdirectivity theory proposed in recent years is attractive, it is hard to implement in practice due to its poor robustness to small random array errors. Hence, in this paper, we present two robust designs of high-order superdirectivity for circular arrays with gain and phase errors. Firstly, we study on the sensitivity function of the high-order superdirectivity and give an alternative solution for a robust superdirective beamformer based on sensitivity function constraint. This method could achieve an arbitrary compromise between directivity and robustness, so it is more flexible and applicable than the existing higher-order truncation method. Although it does not improve on computational complexity or performance with respect to the second-order cone programming obviously, it could lay the foundation for the following robust design method. Then, considering the fact that different eigenbeams correspond to different eigenvalues, we study the method of diagonal loading with variable factors in detail, and further improve the performance of the former sensitivity function constrained method by loading variable factors to different eigenbeams, which results in better performance and greater flexibility in making a compromise. We also show that this proposed loading variable factors method can achieve an equivalent result to the higher-order truncation method by setting proper factors. Simulation results demonstrate the robustness and effectiveness of the above two methods, especially the performance improvement of the loading variable factors method.
\end{abstract}

Keywords: High-order superdirectivity, Circular arrays, Array errors, Sensitivity function constraint, Diagonal loading, Loading variable factors

\section{Introduction}

Beamforming, which is a well-known approach to detect and enhance the desired signal while suppressing noise and interference with a sensor array, has been widely used in many applications $[1,2]$. Among the performance measures of a beamfomer, directivity describes the ability to suppress noise from all directions without affecting the desired signal from one principal direction.

As an optimal design in terms of directivity, superdirectivity [3-5] has attracted tremendous research in different disciplines such as radar [6,7], sonar [8-12], audio engineering [13-16], and wireless communication [17, 18], etc. It is said that a superdirective array with relatively

\footnotetext{
${ }^{*}$ Correspondence: wangmin@nim.ac.cn

'Division of Mechanics and Acoustics, National Institute of Metrology, 100029 Beijing, China
}

Full list of author information is available at the end of the article small size can provide much higher directivity than a conventional array does. In [19] and [20], it was proved that the maximum directivity factor (DF) of an $M$-sensor linear array can reach $M^{2}$ at its endfire direction. It was also proved that the maximum DF of a spherical array is $N^{2}$ with $N$ being the mode number [21].

Compared to the widely used uniform linear array, uniform circular array (UCA) has more compact structure and could form uniform beams over $360^{\circ}$ azimuthal directions. So the superdirectivity of UCA has also attracted considerable attention [8-12, 18]. In [8] and [10], modal beamforming theory was used to analyse the superdirectivity of UCA, but it is not accurate enough due to the inevitable errors caused by spatial sampling and series truncation. Ma recently established a new theory of highorder superdirectivity for UCA [11]. It is based on eigendecomposition of the circulant noise covariance matrix 
and can provide an analytical and closed-form superdirective solution. So it is more accurate and attractive than the modal theory. Then, Wang made some extensions to this high-order superdirectivity theory. He extended it to a general superdirectivity model suitable for arbitrary arrays based on Gram-Schmidt mode-beam decomposition and synthesis [22]. Later, he also provided the analytical optimal solutions for the high-order superdirectivity of circular arrays mounted on a rigid sphere and an infinite rigid cylinder [23].

Although theoretical superdirectivity seems to be very attractive, it is hard to be applied in real-world systems $[5,16,24]$. This is mainly because superdirectivity is very sensitive to the unavoidable random errors of practical arrays, such as sensor gain and phase errors, sensor position perturbations, mutual coupling between sensors, etc. Small deviations can severely degrade the performance of superdirectivity, which may be even worse than that of the conventional beamforming. Thus, robust design methods are eagerly needed to implement superdirectivity in practice.

Diagonal loading method [25] is very useful to improve the robustness, but the problem is that it is not clear how to choose the optimal loading factor. Yan presented a robust supergain beamforming via second-order cone programming (SOCP) [9], and Zhou provided a new superdirective beamforming method to jointly improve array efficiency and robustness for HF circular arrays [18]. But neither of them has provided the robust design solution for the attractive high-order superdirectivity theory. Ma advised to improve the robustness by truncating the sensitive higher-order eigenbeams, i.e., the higher-order truncation (HOT) method [11]. However, this method can only truncate the eigenbeams by a step of certain integral order, so it is not flexible and may be limited in many applications. Wang modified the weighting vector and simplified the diagonal matrix of eigenvalues by virtue of the symmetry properties, then they gave the solution in constraints of both sensitivity function (SF) and sidelobe level by the SOCP [12]. But loading variable factors to different eigenbeams still needs deeper investigation. Wang also discussed the robust design methods for the general superdirectivity model [22] and for the high-order superdirectivity of circular arrays mounted on rigid baffles [23]. Both of them have only used the reduced-rank technique, which is the same with the above HOT method in principle.

In this paper, the robustness of each eigenbeam is investigated in detail for the high-order superdirectivity theory of UCA. Taking SF as the robustness parameter, we firstly give an alternative solution to a constrained superdirective beamformer (CSB) by adding a constraint to SF according to the errors, which is denoted as the CSB method. Although it does not make obvious improvements on computational complexity or performance with respect to SOCP, the CSB method could lay the foundation for a further robust design. Precisely, based on the CSB method and considering that different eigenbeams correspond to different eigenvalues, we present a new design of loading variable factors (LVF) to different eigenbeams, which is named as the LVF method and results in improved performance. It will also be shown that this LVF method could easily achieve an equivalent result to the existing HOT method by setting proper factors.

The remainder of this paper is organised as follows. Firstly, some theory basis of high-order superdirectivity for UCA is given in Sections 2 and 3. Specifically, the theory of high-order superdirectivity is described in Section 2, and its robustness is discussed in Section 3. The interested readers are referred to $[11,12]$ for further details. Then, Section 4 presents the two robust design methods. The performances of the proposed methods are demonstrated by simulation results in Section 5. Finally, the conclusions are summarized in Section 6.

\section{High-order superdirectivity theory}

Consider an $M$-sensor UCA with radius $r$ lying on the $x y$-plane, as shown in Fig. 1. Assuming a unit magnitude plane wave impinging from direction $(\phi, \theta)$, where $\phi$ and $\theta$ are the azimuth and elevation angles, respectively. The pressure received by the $m$ th sensor at $\left(r \cos \phi_{m}, r \sin \phi_{m}, 0\right)$ can be expressed as

$$
p_{m}(\phi, \theta)=e^{j k r \sin \theta \cos \left(\phi_{m}-\phi\right)},
$$

where $k=2 \pi / \lambda$ is the wavenumber with $\lambda$ being the wavelength. Then, the array response vector of the UCA will be

$$
\begin{aligned}
\mathbf{a}(\phi, \theta)= & {\left[e^{j k r \sin \theta \cos \left(\phi_{0}-\phi\right)},\right.} \\
& \left.e^{j k r \sin \theta \cos \left(\phi_{1}-\phi\right)}, \ldots, e^{j k r \sin \theta \cos \left(\phi_{M-1}-\phi\right)}\right]^{\mathrm{T}} \in \mathbb{C}^{M, 1}
\end{aligned}
$$

Suppose the weighting vector and the present steering direction being denoted by $\mathbf{w} \in \mathbb{C}^{M, 1}$ and $\left(\phi_{0}, \theta_{0}\right)$, respectively, the beam pattern (BP) will be

$$
B(\phi, \theta)=\mathbf{w}^{\mathrm{H}}\left(\phi_{0}, \theta_{0}\right) \mathbf{a}(\phi, \theta) .
$$

It is known that solving the optimum weighting vector for the maximum DF is equivalent to maximizing the array gain $G$ in an isotropic noise field $[2,11]$, i.e.,

$$
\mathbf{w}_{\text {opt }}=\arg \max _{\mathbf{w}} \frac{\left|\mathbf{w}^{\mathrm{H}} \mathbf{a}\left(\phi_{0}, \theta_{0}\right)\right|^{2}}{\mathbf{w}^{\mathrm{H}} \boldsymbol{\rho}_{\mathrm{n}} \mathbf{w}},
$$

where $\rho_{\mathrm{n}}$ is the normalized noise covariance matrix in an isotropic noise field. $\rho_{\mathrm{n}}$ is a circulant matrix and its $m$ th eigenvalue and eigenvector are formulated as $\lambda_{m}=$ $\sum_{l=0}^{M-1} \rho_{l} e^{j l \phi_{m}}$ and $\mathbf{v}_{m}=\frac{1}{\sqrt{M}}\left[1, e^{j \phi_{m}}, \ldots, e^{j(M-1) \phi_{m}}\right]^{\mathrm{T}} \in$ 


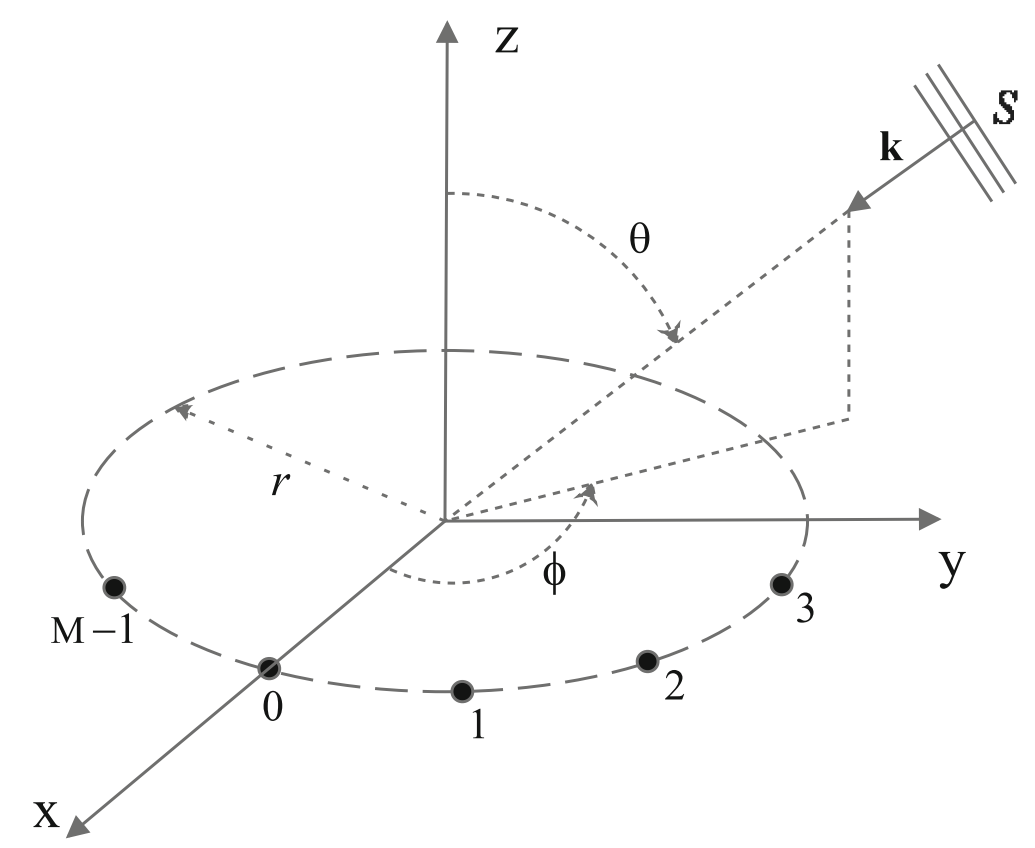

Fig. 1 Geometric model of an M-sensor uniform circular array

$\mathbb{C}^{M, 1}$, respectively [26], where $\rho_{l}=\operatorname{sinc}\left(k \Delta d_{l}\right)$ denotes the $l$ th element of the first row of $\rho_{\mathrm{n}}$ with $\Delta d_{l}$ being the sensor spacing between sensor 0 and sensor $l$ [27]. It is easy to validate that $\lambda_{m}=\lambda_{M-m}$ and $\mathbf{v}_{m}=\mathbf{v}_{M-m}^{*}$.

By using the above property of the circulant symmetric matrix $\boldsymbol{\rho}_{\mathrm{n}}$ and expressing $A_{m}(\phi, \theta)=\mathbf{v}_{m}^{\mathrm{H}} \mathbf{a}(\phi, \theta)$, the closed-form solutions of the optimal weighting vector, the maximum DF and the BP for superdirectivity can be obtained as $[11,12]$

$$
\begin{gathered}
\mathbf{w}_{\mathrm{opt}}=\zeta\left(\sum_{m=0}^{M-1} \frac{1}{\lambda_{m}} \mathbf{v}_{m} \mathbf{v}_{m}^{\mathrm{H}}\right) \mathbf{a}\left(\phi_{0}, \theta_{0}\right), \\
D F_{\max }=G_{\max }=\sum_{m=0}^{M / 2} \frac{\epsilon_{m}}{\lambda_{m}}\left|A_{m}\left(\phi_{0}, \theta_{0}\right)\right|^{2}=\sum_{m=0}^{M / 2} D_{m} \\
B(\phi, \theta)=\zeta \sum_{m=0}^{M / 2} \frac{\epsilon_{m}}{\lambda_{m}} \operatorname{Re}\left\{A_{m}^{*}\left(\phi_{0}, \theta_{0}\right) A_{m}(\phi, \theta)\right\}=\sum_{m=0}^{M / 2} B_{m},
\end{gathered}
$$

where $\zeta=\left[\mathbf{a}^{\mathrm{H}}\left(\phi_{0}, \theta_{0}\right) \boldsymbol{\rho}_{\mathrm{n}}^{-1} \mathbf{a}\left(\phi_{0}, \theta_{0}\right)\right]^{-1}$ is the normalizing coefficient and

$$
\epsilon_{m}= \begin{cases}1 & m=0, M / 2 \\ 2 & m=1,2, \ldots, M / 2-1\end{cases}
$$

Thus, the maximum DF and the optimal BP for superdirectivity of a UCA are decomposed into the sum of the eigenbeams' DFs $D_{m}$ and the sum of the eigenbeams $B_{m}$ ( $m=0,1, \ldots, M / 2)$, respectively. Based on these solutions, a complete theory of high-order superdirectivity for
UCA has been established. More details of this theory can be found in [11].

\section{Analysis of robustness}

Robustness, which measures the beamformer's sensitivity to random errors, is a very crucial point in superdirectivity theory. So it is essential to discuss the robustness of the high-order superdirectivity before our methods are presented.

\subsection{Robustness measurement}

In [11], the eigenvalue $\lambda_{m}$ has been used as the robustness parameter, but no detailed discussions are given. While Wang utilizes the SF to measure the robustness of highorder superdirectivity, since it is directly related to the sum of the variances of array errors and it could provide more information than eigenvalue [12]. We will also take the SF as the robustness measurement in the following discussions. Specifically, the larger the SF is, the poorer the robustness becomes.

The SF of the optimal superdirective beamformer is given by [12]

$T_{\text {sf }}=\left\|\mathbf{w}_{\text {opt }}\right\|^{2}=\sum_{m=0}^{M / 2} \zeta^{2} \frac{\epsilon_{m}}{\lambda_{m}^{2}}\left|A_{m}\left(\phi_{0}, \theta_{0}\right)\right|^{2}=\sum_{m=0}^{M / 2} T_{m}$.

It is observed that the SF can also be decomposed into the sum of eigenbeams' SFs $T_{m}$, just as the maximum DF in (6) and the optimal BP in (7). So we have got both 
the DF $D_{m}$ and the SF $T_{m}$ of each eigenbeam, which are inversely proportional to $\lambda_{m}$ and $\lambda_{m}^{2}$, respectively.

Figure 2 depicts the DFs $D_{m}$ and the SFs $T_{m}$ of various eigenbeams of a 16-sensor UCA both in decibels versus $k r$. It is seen that, for smaller $k r$, the higher the eigenbeam's order is, the larger its DF is, yet the much larger its SF becomes. As $k r$ increases, both the DF and the SF decrease, the superdirective beamformer degrades to the conventional beamformer (CBF). Some related details of the DF and the SF of each eigenbeam are referred to [12].

\subsection{An example}

Providing an 8-sensor UCA with $k r=1$ and $\left(\phi_{0}, \theta_{0}\right)=$ $(\pi / 2, \pi / 2)$, the actual gain $g_{m}$ and phase $\varphi_{m}$ of the $m$ th sensor are chosen according to

$$
g_{m}=1+\sqrt{12} \sigma_{g} \beta_{m}, \quad \varphi_{m}=\sqrt{12} \sigma_{\varphi} \eta_{m}
$$

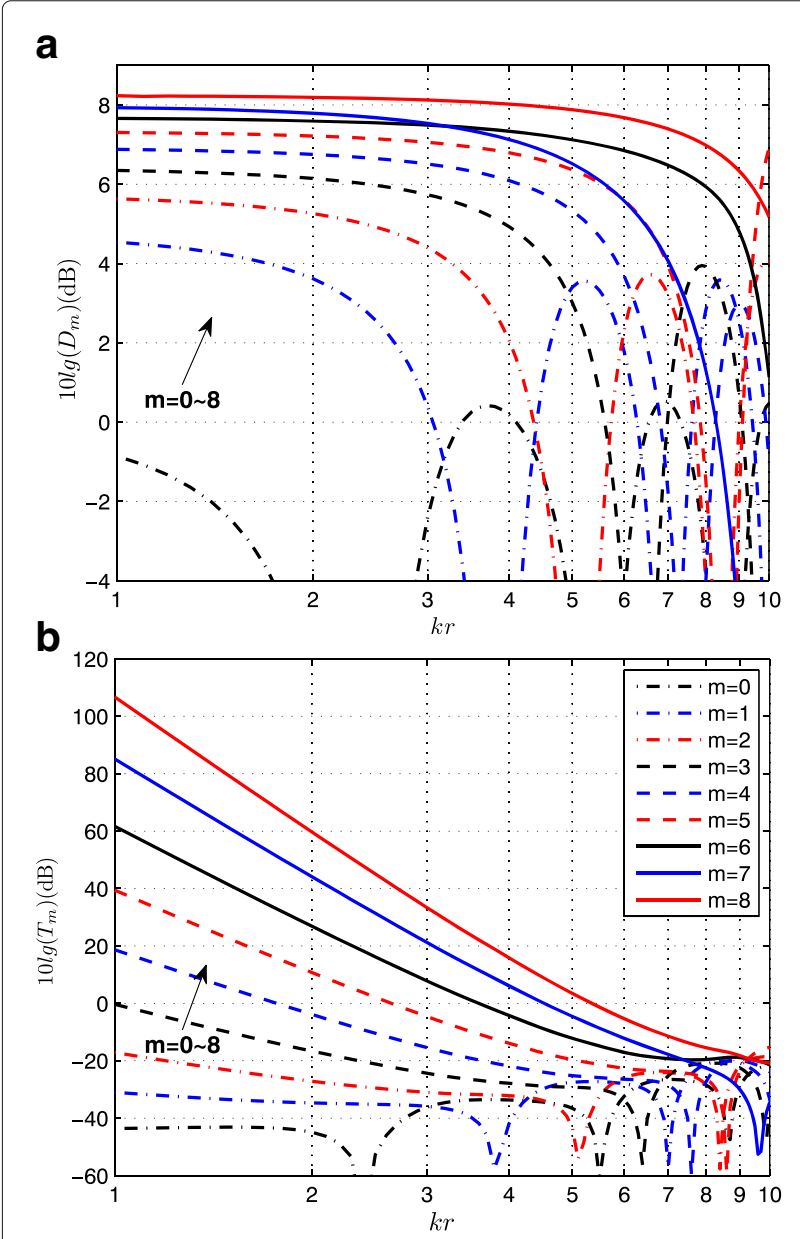

Fig. 2 Directivity factors and sensitivity functions of eigenbeams of a 16-sensor UCA both in decibels versus $k r$. a Directivity factors $10 \lg \left(D_{m}\right)$. b Sensitivity functions $10 \lg \left(T_{m}\right)$ where $\beta_{m}$ and $\eta_{m}$ are i.i.d. random variables in the uniform distribution over $[-0.5,0.5], \sigma_{g}=0.01$ and $\sigma_{\varphi}=1^{\circ}$ are the standard deviations of $g_{m}$ and $\varphi_{m}$, respectively.

The eigenbeams and their synthesized BPs are shown in Fig. 3. For an ideal array without errors, the higher the eigenbeam's order is, the larger its DF or gain is. While for this practical array with errors, some of its higher-order eigenbeams $(m=3,4)$ are distorted obviously, which results in performance degradation of the synthesized BP as seen in Fig. 3f. This example validates the fact that, although the higher-order eigenbeam has higher gains, its robustness is very poor.

A useful way to improve the robustness is the HOT method presented in [11]. However, this method can only truncate the eigenbeams by a step of certain integral order, i.e. the adjustability between the SF and the DF is not flexible. So it may be limited in many applications.

\section{Proposed algorithms}

In this section, we first develop a method of designing the robust high-order superdirectivity based on the SF constraint. This design can be transformed into an extended diagonal loading problem, whose loading factor is directly related to the SF constraint and can be solved by the existing numerical methods. Compared to the HOT method, it can achieve an arbitrary compromise between the superdirective beamformer and the conventional one. Then, although [12] has shown the concept of loading variable factors, it needs study in detail. So, based on the aforementioned high-order superdirectivity theory, we investigate the method of loading variable factors to different eigenbeams, which could result in better performance and be more flexible to make a compromise. Moreover, we will also see that an equivalent result to the HOT method could be achieved by setting proper factors.

\subsection{The CSB method}

Considering that the SF of a superdirective beamformer is undesirably high at low frequencies, we would add a constraint on the SF to make the superdirective beamformer more robust. Denoting the maximum acceptable SF by $\delta_{0}$, the CSB will be

$$
\begin{aligned}
\mathbf{w}_{\mathrm{csb}}= & \arg \min _{\mathbf{w}} \mathbf{w}^{\mathrm{H}} \boldsymbol{\rho}_{\mathrm{n}} \mathbf{w}, \\
& \text { subject to } \mathbf{w}^{\mathrm{H}} \mathbf{a}\left(\phi_{0}, \theta_{0}\right)=1, \quad T_{\mathrm{sf}}=\|\mathbf{w}\|^{2} \leq \delta_{0} .
\end{aligned}
$$

The above equation can also be solved by the SOCP [9]. But considering the fact that this paper focuses on the investigation of loading variable factors to eigenbeams, we provide an alternative solution using a numerical method as follows. 

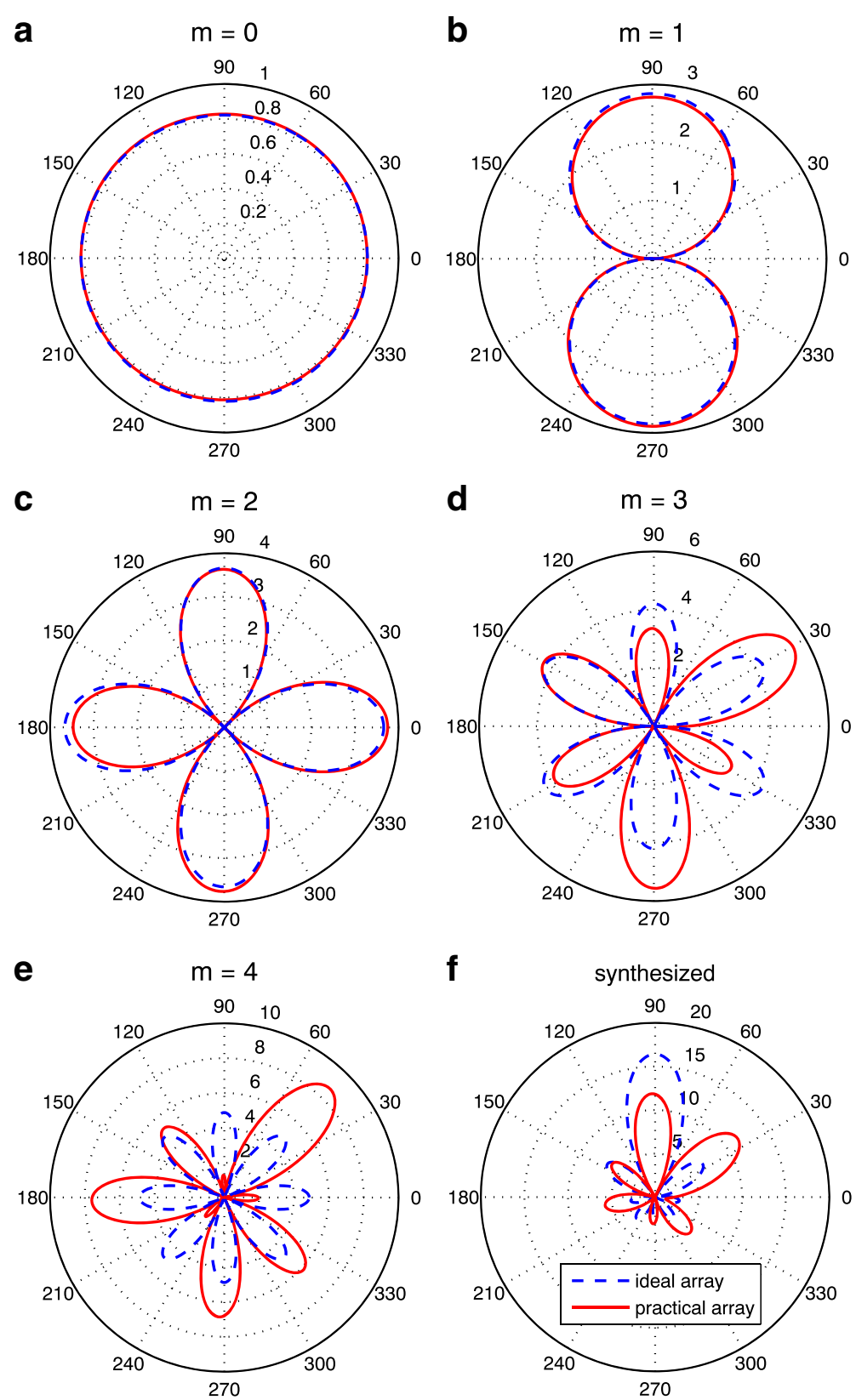

Fig. 3 The 0th- to 4th-order eigenbeams and their synthesized beam patterns for an 8-sensor ideal UCA (blue dashed line) and an 8-sensor practical UCA (red solid line) with $k r=1$ and $\left(\phi_{0}, \theta_{0}\right)=(\pi / 2, \pi / 2)$. a $m=0 . \mathbf{b} m=1 . \mathbf{c} m=2 . \mathbf{d} m=3 . \mathbf{e} m=4$. f Synthesized

The constraint factor $\delta_{0}$ is usually set by designers according to the practical array errors. This could be done by computer simulations or ad hoc experiments simply and efficiently. Specifically, the larger the array errors are, the smaller $\delta_{0}$ should be, thus the more robust the CSB is, yet the smaller the DF is. It is noted that $\delta_{0}$ has a lower bound of $1 / M$ which corresponds to the CBF that is optimal in terms of robustness. In addition, $\delta_{0}$ should be smaller than or equal to the optimal superdirective beamformer's SF $T_{\text {sf }}$ in (9), otherwise the added constraint will be disabled. So the bound of the constraint factor is given by

$$
\frac{1}{M} \leq \delta_{0} \leq \frac{\mathbf{a}^{\mathrm{H}}\left(\phi_{0}, \theta_{0}\right) \boldsymbol{\rho}_{\mathrm{n}}^{-2} \mathbf{a}\left(\phi_{0}, \theta_{0}\right)}{\left[\mathbf{a}^{\mathrm{H}}\left(\phi_{0}, \theta_{0}\right) \boldsymbol{\rho}_{\mathrm{n}}^{-1} \mathbf{a}\left(\phi_{0}, \theta_{0}\right)\right]^{-2}} .
$$

Under the condition of (12), the SF constraint $T_{\mathrm{sf}}=\|$ $\mathbf{w} \|^{2} \leq \delta_{0}$ is enabled and the solution of (11) satisfies $\|$ $\mathbf{w} \|^{2}=\delta_{0}$, then the CSB in (11) can be written as 


$$
\begin{aligned}
\mathbf{w}_{\mathrm{csb}}= & \arg \min _{\mathbf{w}} \mathbf{w}^{\mathrm{H}} \boldsymbol{\rho}_{\mathrm{n}} \mathbf{w}, \\
& \text { subject to } \quad \mathbf{w}^{\mathrm{H}} \mathbf{a}\left(\phi_{0}, \theta_{0}\right)=1, \quad T_{\mathrm{sf}}=\|\mathbf{w}\|^{2}=\delta_{0} .
\end{aligned}
$$

The solution of (13) can be obtained with the method of Lagrange multipliers [28], and is given by

$$
\mathbf{w}_{\mathrm{csb}}=\frac{\left(\boldsymbol{\rho}_{\mathrm{n}}+\gamma \mathbf{I}\right)^{-1} \mathbf{a}\left(\phi_{0}, \theta_{0}\right)}{\mathbf{a}^{\mathrm{H}}\left(\phi_{0}, \theta_{0}\right)\left(\boldsymbol{\rho}_{\mathrm{n}}+\gamma \mathbf{I}\right)^{-1} \mathbf{a}\left(\phi_{0}, \theta_{0}\right)},
$$

where $\gamma$ is a real-valued Lagrange multiplier with $\gamma \geq 0$ and $\left(\boldsymbol{\rho}_{\mathrm{n}}+\gamma \mathbf{I}\right)$ being positive definite, and $\mathbf{I} \in \mathbb{R}^{M, M}$ is a unit matrix. The factor $\gamma$ can be calculated by the following expression which links $\gamma$ to the preset constraint factor $\delta_{0}$ directly,

$\left\|\mathbf{w}_{\mathrm{csb}}\right\|^{2}=\frac{\mathbf{a}^{\mathrm{H}}\left(\phi_{0}, \theta_{0}\right)\left(\boldsymbol{\rho}_{\mathrm{n}}+\gamma \mathbf{I}\right)^{-2} \mathbf{a}\left(\phi_{0}, \theta_{0}\right)}{\left[\mathbf{a}^{\mathrm{H}}\left(\phi_{0}, \theta_{0}\right)\left(\boldsymbol{\rho}_{\mathrm{n}}+\gamma \mathbf{I}\right)^{-1} \mathbf{a}\left(\phi_{0}, \theta_{0}\right)\right]^{2}}=\delta_{0}$.

It is seen from (14) that this design of the CSB belongs to the class of diagonal loading approaches essentially. The main difference between the CSB method and the traditional loading sample matrix inversion (LSMI) method lies in that, it's not clear how to choose the diagonal loading factor of the LSMI method, but the loading factor of our CSB method can be decided by the constraint factor $\delta_{0}$ via Eq. (15).

Under the condition of (12), the middle term in (15) is a monotonically decreasing function of $\gamma$ [28]. Denote this function by $f(\gamma)$, then $f(0)=$ $\mathbf{a}^{\mathrm{H}}\left(\phi_{0}, \theta_{0}\right) \boldsymbol{\rho}_{\mathrm{n}}^{-2} \mathbf{a}\left(\phi_{0}, \theta_{0}\right) /\left[\mathbf{a}^{\mathrm{H}}\left(\phi_{0}, \theta_{0}\right) \boldsymbol{\rho}_{\mathrm{n}}^{-1} \mathbf{a}\left(\phi_{0}, \theta_{0}\right)\right]^{-2}$ and $\lim _{\gamma \rightarrow+\infty} f(\gamma)=1 / M$ just correspond to the upper bound and the lower bound of $\delta_{0}$, respectively. Thus, Eq. (15) has a unique nonnegative solution $\hat{\gamma}$. Considering that (15) is a nonlinear equation, its solution $\hat{\gamma}$ can be obtained efficiently by numerical methods such as a Newton's iterative method.

Next, the upper bound of $\hat{\gamma}$ is worth discussing to avoid the trivial solution [28]. Sorting the eigenvalues $\left\{\lambda_{m}\right\}_{m=0}^{M-1}$ of $\rho_{\mathrm{n}}$ in descending order $\bar{\lambda}_{0} \geq \bar{\lambda}_{1} \geq \ldots \geq \bar{\lambda}_{M-1}$, denoting $\Lambda=\operatorname{diag}\left(\bar{\lambda}_{0}, \bar{\lambda}_{1}, \ldots, \bar{\lambda}_{M-1}\right)$ and the corresponding eigenvectors $\mathbf{U}=\left[\overline{\mathbf{v}}_{0}, \overline{\mathbf{v}}_{1}, \ldots, \overline{\mathbf{v}}_{M-1}\right]$, we can rewrite the matrix $\rho_{\mathrm{n}}$ as

$$
\boldsymbol{\rho}_{\mathrm{n}}=\mathbf{U} \Lambda \mathbf{U}^{\mathrm{H}} \text {. }
$$

Substituting (16) into (15) and utilizing the relationship $\left(\rho_{\mathrm{n}}+\hat{\gamma} \mathbf{I}\right)^{-1}=\mathbf{U}(\Lambda+\hat{\gamma} \mathbf{I})^{-1} \mathbf{U}^{\mathrm{H}}$, we will obtain

$$
\frac{\left(\mathbf{U}^{\mathrm{H}} \mathbf{a}\left(\phi_{0}, \theta_{0}\right)\right)^{\mathrm{H}}(\Lambda+\hat{\gamma} \mathbf{I})^{-2}\left(\mathbf{U}^{\mathrm{H}} \mathbf{a}\left(\phi_{0}, \theta_{0}\right)\right)}{\left[\left(\mathbf{U}^{\mathrm{H}} \mathbf{a}\left(\phi_{0}, \theta_{0}\right)\right)^{\mathrm{H}}(\Lambda+\hat{\gamma} \mathbf{I})^{-1}\left(\mathbf{U}^{\mathrm{H}} \mathbf{a}\left(\phi_{0}, \theta_{0}\right)\right)\right]^{2}}=\delta_{0} \text {. }
$$

Representing the column vector $\mathbf{U}^{\mathrm{H}} \mathbf{a}\left(\phi_{0}, \theta_{0}\right)$ by $\mathbf{z}$ and letting $z_{m}$ denote the $m$ th element of $\mathbf{z}$, (17) can be expanded in element notation

$$
\frac{\sum_{m=0}^{M-1} \frac{\left|z_{m}\right|^{2}}{\left(\sum_{m=0}+\hat{\gamma}\right)^{2}}}{\left[\sum_{m}^{M-1} \frac{\left|z_{m}\right|^{2}}{\left(\bar{\lambda}_{m}+\hat{\gamma}\right)}\right]^{2}}=\delta_{0}
$$

By replacing $\bar{\lambda}_{m}$ in the numerator and the denominator of (18) with $\bar{\lambda}_{M-1}$ and $\bar{\lambda}_{0}$, respectively, we can get an inequality

$\delta_{0} \leq \frac{\frac{\|\mathbf{z}\|^{2}}{\left(\bar{\lambda}_{M-1}+\hat{\gamma}\right)^{2}}}{\frac{\|\mathbf{z}\|^{4}}{\left(\bar{\lambda}_{0}+\hat{\gamma}\right)^{2}}}=\frac{\frac{\left\|\mathbf{a}\left(\phi_{0}, \theta_{0}\right)\right\|^{2}}{\left(\bar{\lambda}_{M-1}+\hat{\gamma}\right)^{2}}}{\frac{\left\|\mathbf{a}\left(\phi_{0}, \theta_{0}\right)\right\|^{4}}{\left(\bar{\lambda}_{0}+\hat{\gamma}\right)^{2}}}=\frac{\left(\bar{\lambda}_{0}+\hat{\gamma}\right)^{2}}{M\left(\bar{\lambda}_{M-1}+\hat{\gamma}\right)^{2}}$,

so the bound of $\hat{\gamma}$ together with $\hat{\gamma} \geq 0$ is given by

$$
0 \leq \hat{\gamma} \leq \frac{\bar{\lambda}_{0}-\bar{\lambda}_{M-1} \sqrt{M \delta_{0}}}{\sqrt{M \delta_{0}}-1}
$$

Finally, summarizing the above design algorithm of the CSB as follows:

1) Perform eigen-decomposition on the normalized noise covariance matrix $\rho_{\mathrm{n}}$.

2) Set a proper value for the constraint factor $\delta_{0}$ according to array errors. If (12) is satisfied, solve (18) for $\hat{\gamma}$ by a Newton's iterative method under the condition of (20); otherwise, set $\hat{\gamma}=0$.

3) Substitute the $\hat{\gamma}$ obtained from Step 2 into (14) or

$$
\mathbf{w}_{\mathrm{csb}}=\frac{\mathbf{U}(\Lambda+\gamma \mathbf{I})^{-1} \mathbf{U}^{\mathrm{H}} \mathbf{a}\left(\phi_{0}, \theta_{0}\right)}{\mathbf{a}^{\mathrm{H}}\left(\phi_{0}, \theta_{0}\right) \mathbf{U}(\Lambda+\gamma \mathbf{I})^{-1} \mathbf{U}^{\mathrm{H}} \mathbf{a}\left(\phi_{0}, \theta_{0}\right)}
$$

to calculate the desired weighting vector of the CSB.

It is noteworthy that both the SOCP and the CSB methods require a complexity of $O\left(M^{3}\right)[9,28]$ and they can achieve the similar performance. But the CSB method could lay the foundation for the following LVF method and also facilitate the sequent comparison and analysis, so it is more suitable for the robust high-order superdirectivity in this paper.

\subsection{The LVF method}

The above method achieves compromise between the DF and the SF by loading the same factor $\hat{\gamma}$ to all the eigenvalues. Considering that the eigenvalues of high-order superdirectivity vary within a very wide range, e.g., the maximum eigenvalue $\bar{\lambda}_{0}=5.703$ and the minimum eigenvalue $\bar{\lambda}_{4}=(4.025 \mathrm{e}-5)$ of an 8 -sensor UCA with $k r=1$, we would expect that loading variable factors to different eigenvalues which correspond to different eigenbeams will improve the above method to some extent.

Denoting the different loading factors by $\left\{\hat{\gamma}_{m}\right\}_{m=0}^{M-1}$ and using the original eigenvalues $\lambda_{m}$ instead of the sorted ones $\bar{\lambda}_{m}$ for convenience, we can modify (21) as 


$$
\mathbf{w}_{\mathrm{lvf}}=\frac{\mathbf{U} \hat{\Lambda}^{-1} \mathbf{U}^{\mathrm{H}} \mathbf{a}\left(\phi_{0}, \theta_{0}\right)}{\mathbf{a}^{\mathrm{H}}\left(\phi_{0}, \theta_{0}\right) \mathbf{U} \hat{\Lambda}^{-1} \mathbf{U}^{\mathrm{H}} \mathbf{a}\left(\phi_{0}, \theta_{0}\right)},
$$

where $\hat{\Lambda}=\operatorname{diag}\left(\lambda_{0}+\hat{\gamma}_{0}, \lambda_{1}+\hat{\gamma}_{1}, \ldots, \lambda_{M-1}+\hat{\gamma}_{M-1}\right)$. These loading factors can be decided independently according to the eigenbeams' robustness. Proper choosing of each loading factor will achieve a better compromise between the DF and the SF of a superdirective beamformer.

Further, from Eqs. (6), (7), and (9), we can see that for a very small eigenvalue $\lambda_{m}$ which corresponds to a highorder eigenbeam, its inverse $1 / \lambda_{m}$ is infinitely large, so that even small array errors may be greatly amplified. In this case, robustness is the main factor and we do not expect an accurate approximation of $1 / \lambda_{m}$, so a larger loading factor $\hat{\gamma}^{2} / \lambda_{m}$ is more proper than $\hat{\gamma}$. While for a large eigenvalue $\lambda_{m}$, its eigenbeam is so robust against array errors that there is no need to load any factor. And it can be easily seen that $\left(\lambda_{m}+\hat{\gamma}^{2} / \lambda_{m}\right)$ is closer to $\lambda_{m}$ than $\left(\lambda_{m}+\hat{\gamma}\right)$ with an increasing eigenvalue. Thus a loading factor which varies with its eigenvalue seems to be more reasonable than a fixed one.

Specifically, we propose to relate the $m$ th loading factor $\hat{\gamma}_{m}$ with its corresponding eigenvalues $\lambda_{m}$ and the aforementioned solution $\hat{\gamma}$, i.e.,

$$
\hat{\gamma}_{m}=\frac{\hat{\gamma}^{2}}{\lambda_{m}}, \quad m=0,1, \ldots, M-1 .
$$

The matrix $\rho_{\mathrm{n}}$ will become $\mathbf{R}_{\mathrm{n}}=\left(\boldsymbol{\rho}_{\mathrm{n}}+\Lambda_{\mathrm{VL}}\right)$, where $\Lambda_{\mathrm{VL}}=\operatorname{diag}\left(\hat{\gamma}_{0}, \hat{\gamma}_{1}, \ldots, \hat{\gamma}_{M-1}\right)$. Together with the modified matrix $\mathbf{G}_{\mathrm{n}}=\left(\boldsymbol{\rho}_{\mathrm{n}}+\hat{\gamma} \mathbf{I}\right)$ in the above CSB method, the three matrices $\boldsymbol{\rho}_{\mathrm{n}}, \mathbf{G}_{\mathrm{n}}$ and $\mathbf{R}_{\mathrm{n}}$ have the same eigenvectors and different eigenvalues $\lambda_{m},\left(\lambda_{m}+\hat{\gamma}\right)$ and $\left(\lambda_{m}+\hat{\gamma}^{2} / \lambda_{m}\right)$, respectively. The performances of these two methods will be demonstrated in Section 5 by simulation results.

It should be noted that Eq. (23) is mainly taken as one example to show the potential improved performance of the LVF method. The analytical optimal solution for variable factors $\left\{\hat{\gamma}_{m}\right\}_{m=0}^{M-1}$ still needs more investigation, and it would be one of our main future research tracks.

Before closing this section, we will show the relationship between this LVF method and the aforementioned HOT method. From (6) and (9), the DF and the SF of the $m$ thorder eigenbeam with a variable loading factor $\hat{\gamma}_{m}$ can be respectively expressed as

$$
\hat{D}_{m}=\frac{\epsilon_{m}\left|A_{m}\left(\phi_{0}, \theta_{0}\right)\right|^{2}}{\lambda_{m}+\hat{\gamma}_{m}}
$$

and

$$
\hat{T}_{m}=\zeta^{2} \frac{\epsilon_{m}\left|A_{m}\left(\phi_{0}, \theta_{0}\right)\right|^{2}}{\left(\lambda_{m}+\hat{\gamma}_{m}\right)^{2}}
$$

where $m=0,1, \ldots, M / 2$. Only $(M / 2+1)$ loading factors are considered since $\lambda_{m}=\lambda_{M-m}$.

In (24) and (25), two special values for $\hat{\gamma}_{m}$ are worth discussing. One is $\hat{\gamma}_{m}=+\infty$, then the DF $\hat{D}_{m}$ and the SF $\hat{T}_{m}$ both equal 0 , which means the $m$ th-order eigenbeam is not available, or i.e., truncated. The other is $\hat{\gamma}_{m}=0$, thus the DF $\hat{D}_{m}$ and the SF $\hat{T}_{m}$ keep unchanged with their counterparts in the high-order superdirectivity theory, so that the $m$ th-order eigenbeam will be reserved. In conclusion, the result of the HOT method, which can also be implemented by setting $\hat{\gamma}_{m}=0$ to the lower-order eigenbeams and $\hat{\gamma}_{m}=+\infty$ to the higher-order eigenbeams, is contained in our LVF method.

\section{Simulation results}

In this section, some simulation results are presented to illustrate the performances of the proposed methods. Firstly, the design of the CSB is shown with several different SF constraint factors. Then, the performances of the CSB method and the LVF method are compared by a design example. At last, the relationship between the LVF method and the HOT method is shown and discussed. Without loss of generality, the present steering direction is set as $\left(\phi_{0}, \theta_{0}\right)=(\pi / 2, \pi / 2)$ in the following simulations.

\subsection{Simulations of the CSB method}

Consider a 16-sensor UCA with $k r$ ranging from 1 to 15 , the SF constraint factor $\delta_{0}$ is set as $80,60,40,20$, and 0 $\mathrm{dB}$, respectively. The total DF (or its logarithmic form the directivity index (DI), which is defined as DI $=10 \lg (D F)$ $(\mathrm{dB})$ ) and SF of the CSB are depicted in Fig. 4. The conventional and ideal superdirective results are added for comparison, where the conventional result is obtained by using the weighting vector $\mathbf{w}=\mathbf{a}\left(\phi_{0}, \theta_{0}\right) / M$, and the ideal superdirective result is obtained by using the optimal weighting vector in (5). Some relevant conclusions can be made as follows.

It is shown from Fig. 4 that, for the ideal superdirective beamformer, its DF decreases and its SF drops more quickly as $k r$ increases, it will finally degrade to the conventional beamformer since the normalized noise covariance matrix $\rho_{\mathrm{n}}$ tends to a unit matrix. These results have just validated the fact that superdirectivity can only be achieved for small $k r$ [13].

In Fig. $4 \mathrm{~b}$, the SF of the proposed CSB is seen to be constrained less than or equal to the SF constraint factor $\delta_{0}$, while the corresponding DF decreases to a certain extent, as shown in Fig. 4a. The smaller the SF constraint factor $\delta_{0}$ is, the more robust the CSB becomes, but the smaller the DF is. It is validated that the CSB method could make a flexible compromise between the superdirective beamformer and the conventional one, thus it is more useful to practical circular arrays with errors. 


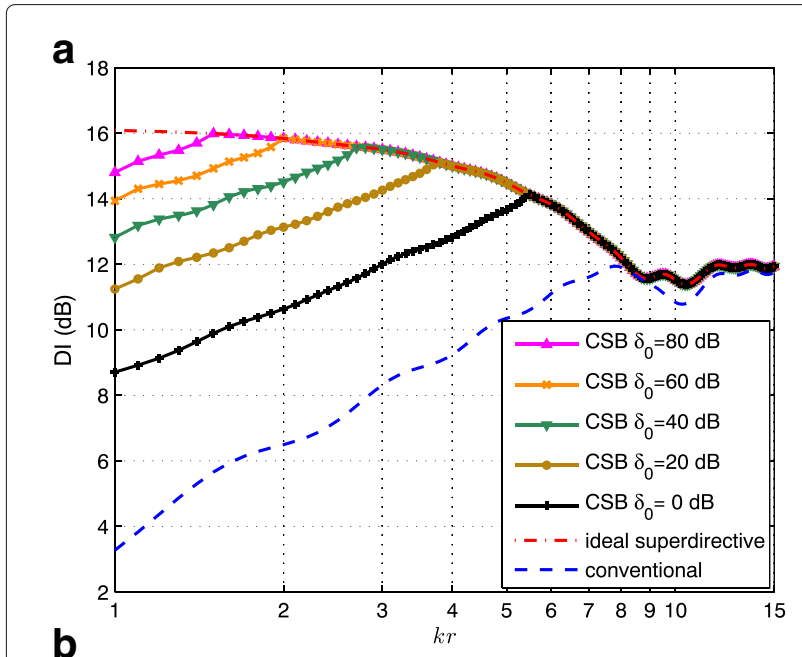

b

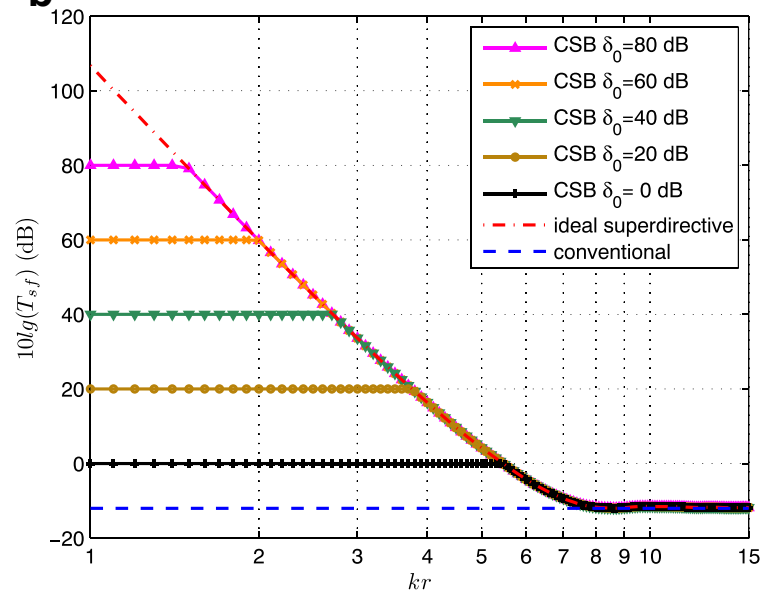

Fig. 4 Total directivity index and sensitivity function of the CSB versus $k r$ with various constraint factors. a Directivity index. b Sensitivity function

It should be noted that, if (12) is satisfied, the constraint factor $\delta_{0}$ is effective and equal to the SF of the CSB; but when $\delta_{0}$ is larger than the SF of superdirectivity as $k r$ increases, it will be ineffective, then the CSB is equivalent to the superdirective beamformer, as shown in Fig. 4.

\subsection{Comparisons of the CSB and LVF methods}

To compare the performances of the CSB and LVF methods, we set $\delta_{0}=20 \mathrm{~dB}$ and $M=12$. The loading factors $\hat{\gamma}$ and $\left\{\hat{\gamma}_{m}\right\}_{m=0}^{M-1}$ are, respectively, computed by (18) and (23). The total DI (or DF in decibel) and SF are shown in Fig. 5.

It is seen from Fig. $5 \mathrm{~b}$ that, for small $k r$, the SF of the CSB is constrained to be $\delta_{0}$ while the SF of the LVF is variable and lower than $\delta_{0}$ at most points. Meanwhile, the DFs of the LVF and the CSB remain almost the same level, as shown in Fig. 5a. As $k r$ increases, both the CSB and LVF methods become the superdirective beamformer and tend to the conventional one finally. These results

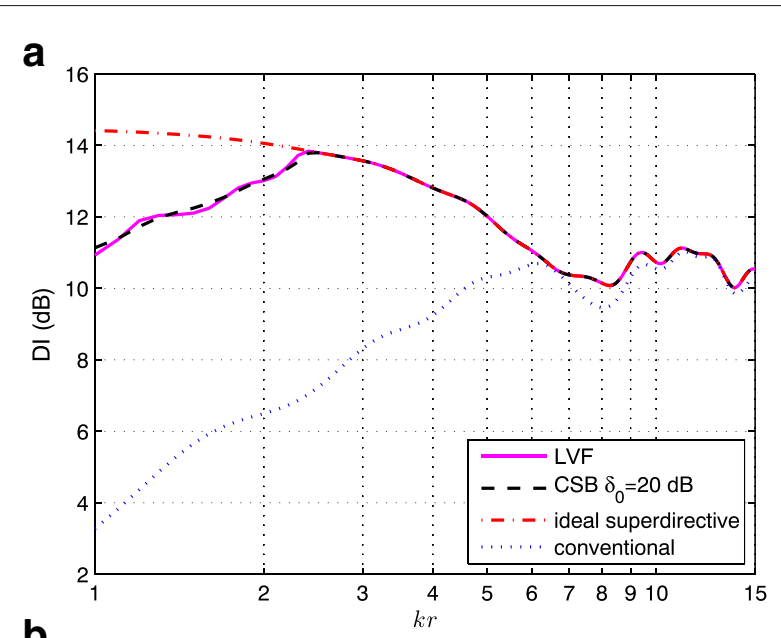

b

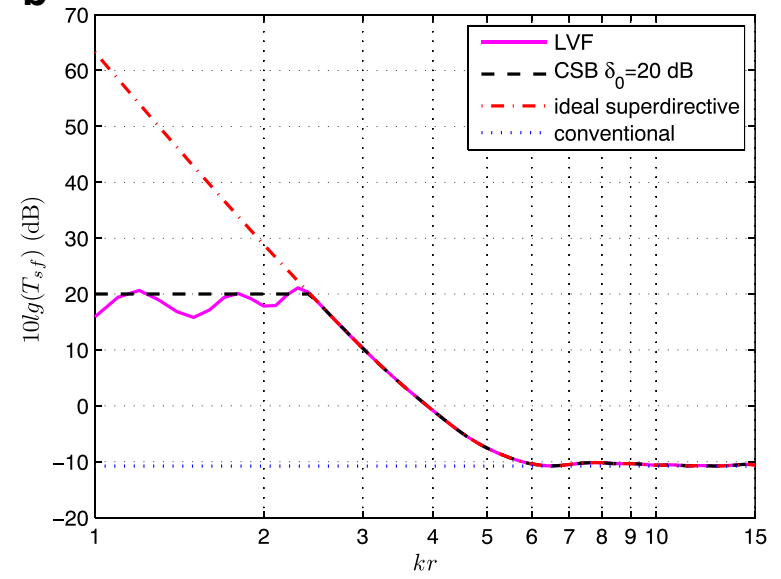

Fig. 5 Total directivity index (or directivity factor in decibel) and sensitivity function of the CSB and the LVF versus $k r$ for a 12 -sensor UCA. a Directivity index. b Sensitivity function

show that the LVF method could achieve a more flexible compromise than the CSB method between superdirectivity and robustness. Moreover, the LVF method is somewhat more robust for most small $k r$.

Next, we add some random gain and phase errors to the 12-sensor array with $k r=1.5$ according to (10) with $\sigma_{g}=0.01$ and $\sigma_{\varphi}=1^{\circ}$, and examine the eigenbeams and synthesized BPs of four cases (superdirectivity of the practical array with errors, superdirectivity of the ideal array without errors, CSB design with array errors, and LVF design with array errors).

Figure 6 shows the 2nd- to 6th-order eigenbeams of the above four cases. The 0th- and 1st-order eigenbeams are not given here since they are robust enough. Figure $6 f$ is a magnification of a portion of Fig. 6e. It can be seen that the lower-order eigenbeams of the four cases are almost consistent. As the order increases, the three cases with array errors deviate from the ideal case. Especially, the 5th- and 6th-order eigenbeams of the practical array are 

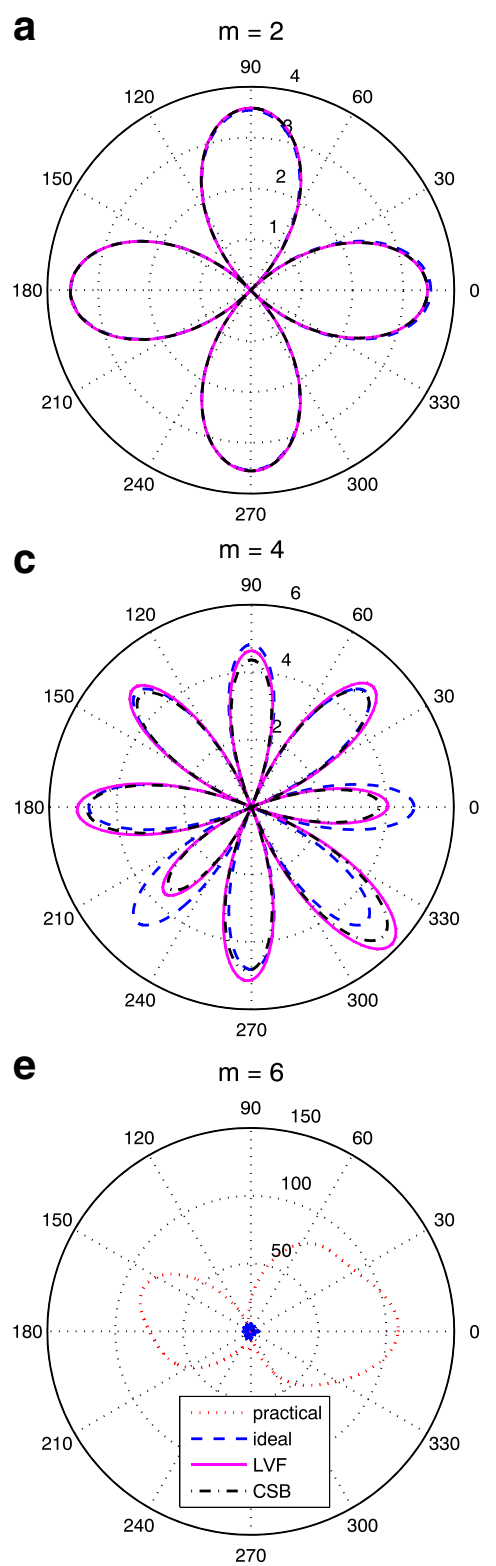
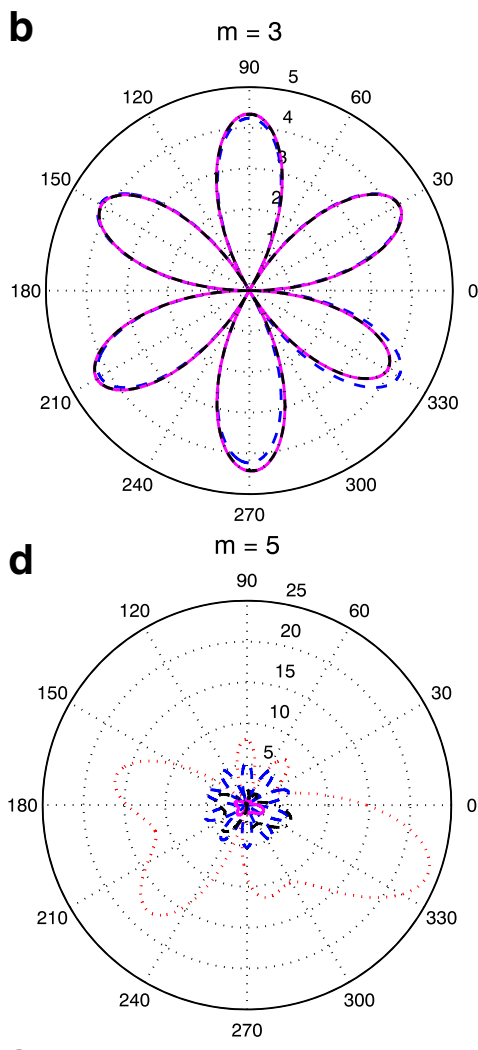

f

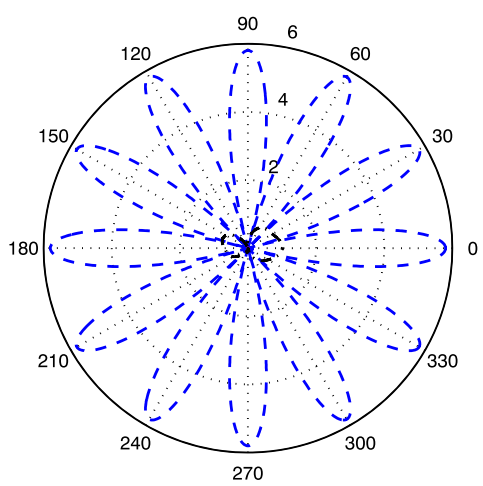

Fig. 6 The 2nd- to 6th-order eigenbeams of four cases (practical array, ideal array, LVF design and CSB design) for a 12-sensor array with $\mathrm{kr}=1.5 \mathrm{in}$ the presence of array gain and phase errors. $\mathbf{a} m=2 . \mathbf{b} \mathrm{m}=3 . \mathbf{c} \mathrm{m}=4$. $\mathbf{d} \mathrm{m}=5$. e $\mathrm{m}=6$. $\mathbf{f}$ Magnification of a portion of $\mathbf{e}$

seriously distorted due to their poor robustness, while these two eigenbeams of the CSB and LVF methods are significantly reduced, especially the LVF case. That is why the robustness could be improved by using the CSB and LVF methods.

Figure 7 depicts the total BPs of the four cases. Again, Fig. $7 \mathrm{~b}$ is a magnification of a portion of Fig. $7 \mathrm{a}$ with the $\mathrm{CBF}$ for comparison. The superdirective BP of the practical array with gain and phase errors (labelled as "practical" in Fig. 7a), which is synthesized using all practical eigenbeams from order 0 to order 6 , is shown to be extremely distorted. But the BPs of the two proposed methods CSB and LVF are greatly improved. They also achieve higher DFs and narrower mainlobe widths than the CBF does. Although their DFs are smaller than the ideal case, they are more robust and more proper for a practical circular array with gain and phase errors. From Fig. 7b, we can also obtain that the mainlobe of the LVF method is very approximate to the one of the CSB method. But the sidelobe levels of the CSB and LVF methods are about -5.6 and $-6.66 \mathrm{~dB}$, respectively, i.e., the LVF method has an improvement of $1.06 \mathrm{~dB}$ in terms of sidelobel level than 
a

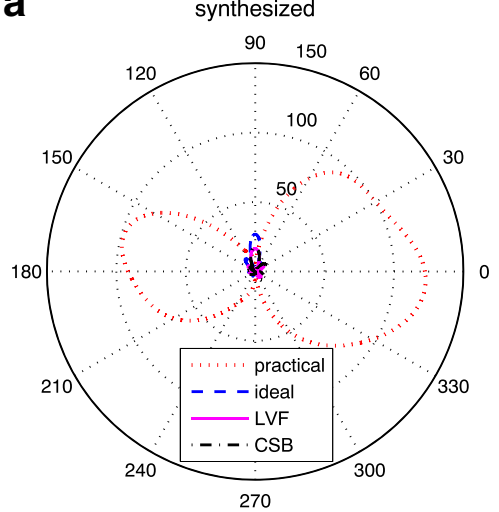

b

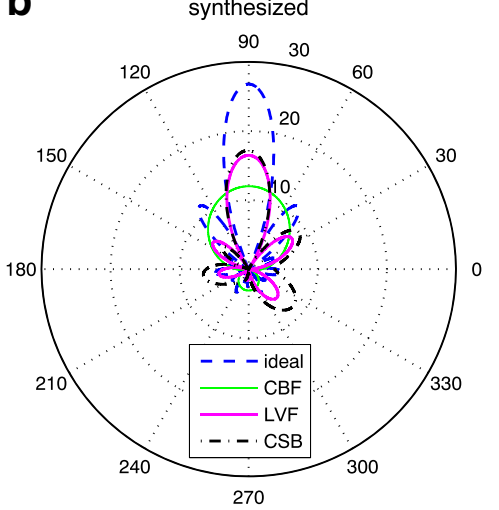

Fig. 7 Comparison of total beam patterns with gain and phase errors for a 12-sensor UCA, $k r=1.5$. a Total beam patterns without including CBF. $\mathbf{b}$ Magnification of a portion of a with CBF

the CSB method. In addition, the BP of the LVF method is more symmetrical and its shape is closer to the ideal BP, which means that the LVF method is more robust to gain and phase errors than the CSB method.

To compare the performance of the two methods further, we change the simulation conditions with $M=16$, $k r=1.65$ and $\delta_{0}=30 \mathrm{~dB}$. The levels of gain and phase errors keep unchanged. The simulation results of the total BPs are shown in Fig. 8. We can see that the performance of the BP of the LVF method outperforms that of the CSB method obviously. Although its mainlobe is somewhat widened, the sidelobe level and the shape of the BP of the LVF method are much improved, while the BP of the CSB method is relatively poor in performance.

At last, comparing the results of Figs. $7 \mathrm{~b}$ and $8 \mathrm{a}$, we can find that the DF achieved by the LVF method is better than that of the CSB method in Fig. 8a, while it is somewhat worse in Fig. 7b. The main reason lies in that the BPs of the two methods are distorted in varying degrees by array errors. In Fig. 7b, the SF constraint factor $\delta_{0}$ is $20 \mathrm{~dB}$, so both the BPs of the two methods are relatively less distorted. The DF of the LVF method is a little worse than that of the CSB method, but the LVF method is shown to be more robust. This is reasonable because the DF and the SF are a pair of tradeoffs theoretically. While in Fig. 8a, $\delta_{0}$ is increased to $30 \mathrm{~dB}$ and the array errors play a main role in this case. The two BPs are distorted more seriously, especially the CSB method. So the DF of the LVF method is better because its BP is less affected by array errors than that of the CSB method.

\subsection{The LVF method versus the HOT method}

In the last simulation, we will illustrate the relationship between our LVF method and the existing HOT method.

Reconsider a 12-sensor UCA, and set the loading factors $\hat{\gamma}_{m}$ as $+\infty$ for $m=5,6,7$, and 0 for the others,
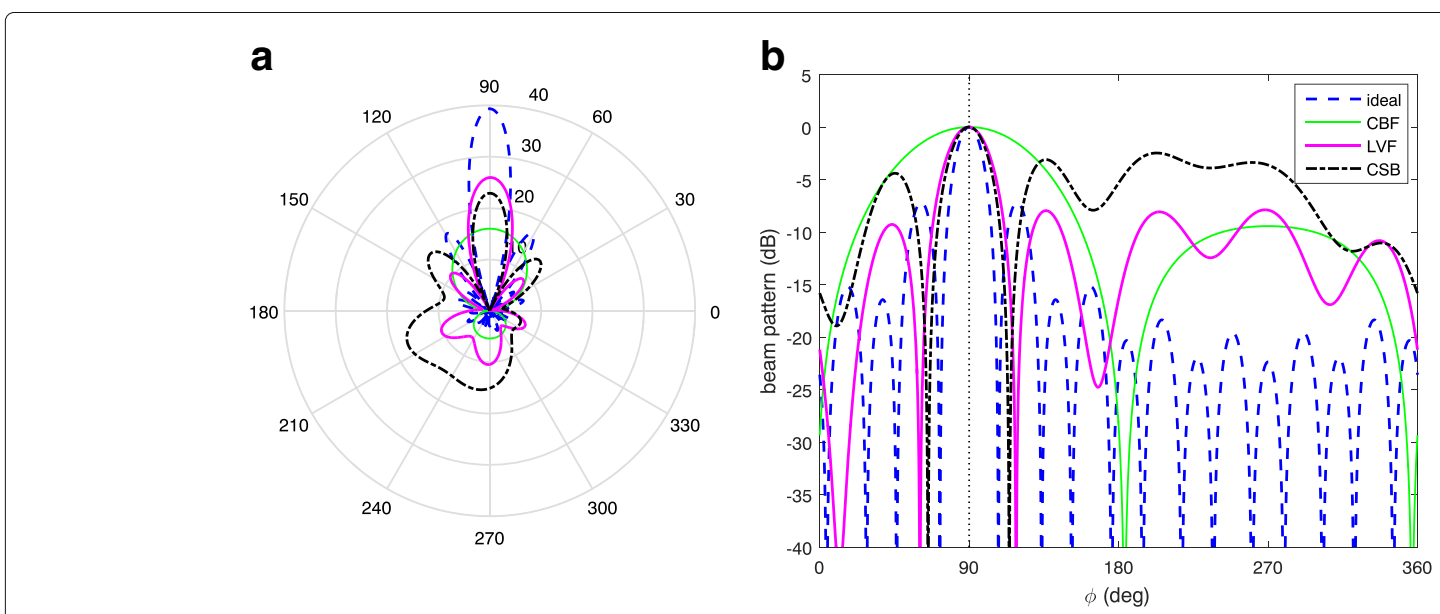

Fig. 8 Comparison of total beam patterns with gain and phase errors for a 16-sensor UCA with $k r=1.65, \delta_{0}=30 \mathrm{~dB}$. a Polar coordinates figure. $\mathbf{b}$ Normalized beam patterns 
respectively. Actually, this is equivalent to loading $+\infty$ to the 5th- and 6th-order eigenbeams and loading 0 to the 0th- to 4th-order ones since $\lambda_{m}=\lambda_{M-m}$. On the other hand, truncate the 5th- and 6th-order eigenbeams directly using the HOT method. The corresponding total DI (or DF in decibel) and SF are shown in Fig. 9a and $\mathrm{b}$, respectively. It can be seen that both of the DFs and SFs of the two methods match well with each other. So our LVF method could obtain an equivalent result to the HOT method by properly setting 0 and $+\infty$ to the loading factors.

Then, under the condition of the aforementioned array errors with $k r=1.5$, we design the HOT method by synthesizing the 0th- to 5th-order eigenbeams and the 0th- to 4th-order eigenbeams, respectively, and design the LVF method by setting $\delta_{0}=20 \mathrm{~dB}$. The BPs of the three cases are shown in Fig. 9c. It is seen that the LVF method has the similar BP to the HOT $(0 \sim 4)$ method. Although its mainlobe width is about $7^{\circ}$ broader than that of the $\operatorname{HOT}(0 \sim 5)$ method, its sidelobe level (about $-6.66 \mathrm{~dB}$ ) is very much improved since the BP of the $\operatorname{HOT}(0 \sim 5)$ method is severely distorted and useless. Further, we increase the constraint factor of the LVF method to $\delta_{0}=23 \mathrm{~dB}$ in Fig. 9d. Compared with the $\operatorname{HOT}(0 \sim 4)$ method, the mainlobe width of the LVF method is reduced by about $2.5^{\circ}$, but its sidelobe level is increased by about $0.5 \mathrm{~dB}$. Moreover, the LVF method is still much more robust than the $\operatorname{HOT}(0 \sim 5)$ method. These results show the fact that the LVF method could adjust the compromise between directivity and robustness more flexibly.

Thus, our LVF method could fill the gap between different eigenbeams truncations of the HOT method, and it is more flexible to make a compromise and has the potential to provide better performance in the robust design of high-order superdirectivity.

\section{Conclusions}

In conclusion, we make a detailed study of the variable loading method for improving the robustness of highorder superdirectivity for circular arrays. Since higherorder eigenbeams are very sensitive to array gain and phase errors, the theoretical superdirectivity is hardly implemented in practice. To solve this problem, we present two robust design methods in this paper. Firstly, by adding a constraint to the SF, a more robust CSB is produced which can achieve a compromise between the superdirective beamformer and the conventional one, in terms of directivity and robustness. Further, a more flexible LVF method is proposed by loading variable factors to

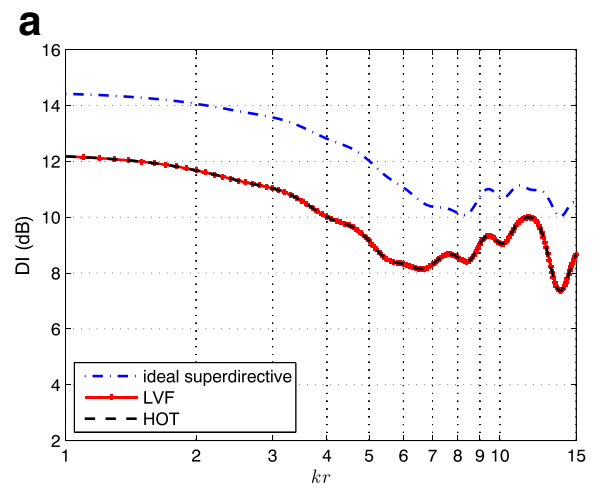

C

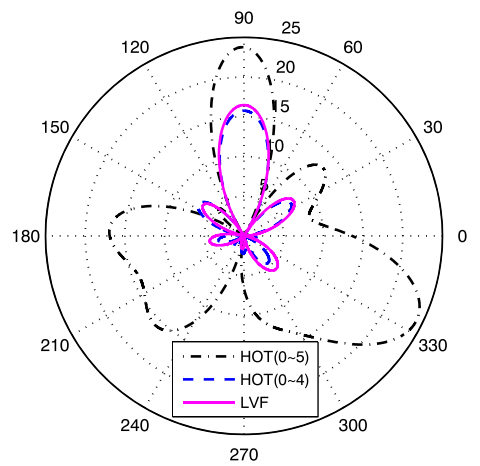

b

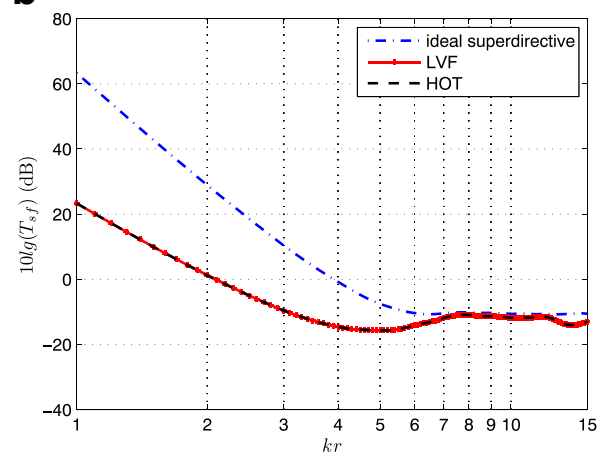

d

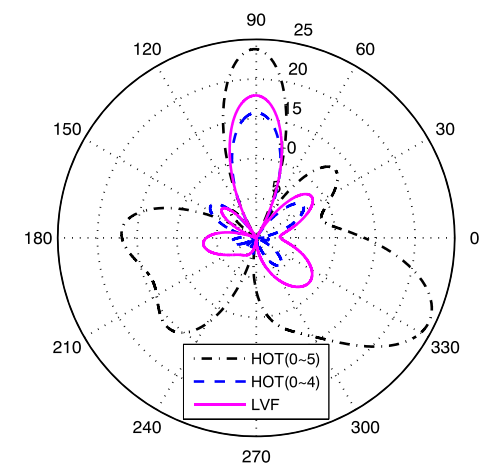

Fig. 9 Comparison of the loading variable factors method and the higher-order truncation method. a Total directivity index and $\mathbf{b}$ total sensitivity function $10 \lg \left(T_{\text {sf }}\right)$ of the three beamformers. Beam patterns of the HOT method and the LVF method with $\mathbf{c} \delta_{0}=20 \mathrm{~dB}$, and $\mathbf{d} \delta_{0}=23 \mathrm{~dB}$ 
different eigenbeams, and it has better performance than the CSB method at most frequencies. We also prove that the LVF method contains the results of the existing HOT method and could outperform it. Simulation results have demonstrated that the robustness of high-order superdirectivity to gain and phase errors is obviously improved by the proposed methods. The effects of other factors on high-order superdirectivity, such as mutual coupling and array efficiency, still need more investigation, and it would be our another main future research track.

\section{Acknowledgements}

The authors would like to thank the anonymous reviewers for their insightful comments and suggestions, which helped improve the quality of this paper significantly.

\section{Funding}

This work was supported by the National Natural Science Foundation of China under Grants 51505453, 61471352 and 61571434.

\section{Authors' contributions}

MW and XCM conceived the basic idea and designed the numerical simulations. MW and PY analyzed the simulation results. CPH analyzed derivation of equations and revised the manuscript. XJF and $Y Z$ checked the simulations and refined the whole manuscript. MW wrote and revised the manuscript. All authors read and approved the final manuscript.

\section{Competing interests}

The authors declare that they have no competing interests.

\section{Author details}

${ }^{1}$ Division of Mechanics and Acoustics, National Institute of Metrology, 100029 Beijing, China. ${ }^{2}$ Institute of Acoustics, Chinese Academy of Sciences, 100190 Beijing, China.

Received: 16 August 2016 Accepted: 14 February 2017

Published online: 21 February 2017

\section{References}

1. H Krim, M Viberg, Two decades of array signal processing research: the parametric approach. IEEE Signal Proc. Mag. 13, 67-94 (1996)

2. HL Van Trees, Optimum Array Processing: Part IV of Detection, Estimation, and Modulation Theory. (Wiley, New York, 2002), pp. 59-71, 274-289, 439-443

3. EH Newman, JH Richmond, $\mathrm{CH}$ Walter, Superdirective receiving arrays. IEEE Trans. Antennas Propag. 26(5), 629-635 (1978)

4. M Crocco, A Trucco, Design of robust superdirective arrays with a tunable tradeoff between directivity and frequency-invariance. IEEE Trans. Signal Process. 59, 2169-2181 (2011)

5. A Trucco, M Crocco, Design of an optimum superdirective beamformer through generalized directivity maximization. IEEE Trans. Signal Process. 62, 6118-6129 (2014)

6. DP Scholnik, JO Coleman, in IEEE Radar Conf. Superdirectivity and SNR constraints in wideband array-pattern design (IEEE, Atlanta, 2001), pp. 181-186

7. ML Morris, MA Jensen, JW Wallace, Superdirectivity in MIMO systems. IEEE Trans. Antennas Propag. 53(9), 2850-2857 (2005)

8. JB Franklin, Superdirective receiving arrays for underwater acoustics application. (DREA Report No. CR/97/444, Defence Research Establishment Atlantic, Dartmouth, Nova Scotia, 1997)

9. SF Yan, YL Ma, Robust supergain beamforming for circular array via second-order cone programming. Appl. Acoust. (Elsevier). 66(9), 1018-1032 (2005)

10. YX Yang, W Jiang, YL Ma, in OCEANS 2007. Phased-mode circular multi-channel hydrophone with super directivity (IEEE, Vancouver, 2007), pp. 1-5

11. YL Ma, YX Yang, ZY He, KD Yang, C Sun, YM Wang, Theoretical and practical solutions for high-order superdirectivity of circular sensor arrays. IEEE Trans. Ind. Electron. 60, 203-209 (2013)
12. Y Wang, YX Yang, YL Ma, ZY He, Robust high-order superdirectivity of circular sensor arrays. J. Acoust. Soc. Am. 136(4), 1712-1724 (2014)

13. J Bitzer, KU Simmer, in Microphone arrays: Signal Processing Techniques and Applications. Superdirective microphone arrays (Springer-Verlag, New York, 2001), pp. 19-38

14. TD Abhayapala, DB Ward, in IEEE International Conference On Acoustics, Speech and Signal Processing (ICASSP). Theory and design of high order sound field microphones using spherical microphone array (IEEE, Florida, 2002), pp. 1949-1952

15. TD Abhayapala, A Gupta, Spherical harmonic analysis of wavefields using multiple circular sensor arrays. IEEE Trans. Audio Speech Lang. Process. 18(6), 1655-1664 (2010)

16. S Doclo, M Moonen, Superdirective beamforming robust against microphone mismatch. IEEE Trans. Audio Speech Lang. Process. 15(2), 617-631 (2007)

17. NW Bikhazi, MA Jensen, The relationship between antenna loss and superdirectivity in MIMO systems. IEEE Trans. Wirel. Commun. 6(5), 1796-1802 (2007)

18. Q-C Zhou, HT Gao, HJ Zhang, F Wang, Robust superdirective beamforming for hf circular receive antenna arrays. Prog. Electromagn. Res. 136, 665-679 (2013)

19. Al Uzkov, An approach to the problem of optimum directive antenna design. Compt. Rend. Acad. Sci. U.R.S.S. 53, 35-38 (1946)

20. AT Parsons, Maximum directivity proof for three-dimensional arrays. J. Acoust. Soc. Am. 82(1), 179-182 (1987)

21. JL Butler, SL Ehrlich, Superdirective spherical radiator. J. Acoust. Soc. Am. 61(6), 1427-1431 (1977)

22. Y Wang, YX Yang, ZY He, YN Han, YL Ma, A general superdirectivity model for arbitrary sensor arrays. EURASIP J. Adv. Signal Process. 2015(1), 1-16 (2015)

23. Y Wang, YX Yang, YL Ma, ZY He, High-order superdirectivity of circular sensor arrays mounted on baffles. Acta Acust. united Ac. 102(1), 80-93 (2016)

24. H Cox, RM Zeskind, T Kouij, Practical supergain. IEEE Trans. Acoust. Speech Signal Process. 34(3), 393-398 (1986)

25. BD Carlson, Covariance matrix estimation errors and diagonal loading in adaptive arrays. IEEE Trans. Aero. Elec. Syst. 24(3), 397-401 (1988)

26. RM Gray, Toeplitz and circulant matrices: a review. Found. Trends Commun. Inf. Theory. 2(3), 155-239 (2006)

27. BF Cron, CH Sherman, Spatial-correlation functions for various noise models. J. Acoust. Soc. Am. 34(11), 1732-1736 (1962)

28. J Li, P Stoica, ZS Wang, Doubly constrained robust Capon beamformer. IEEE Trans. Signal Process. 52(9), 2407-2423 (2004)

\section{Submit your manuscript to a SpringerOpen ${ }^{\circ}$ journal and benefit from:}

- Convenient online submission

- Rigorous peer review

- Immediate publication on acceptance

- Open access: articles freely available online

- High visibility within the field

- Retaining the copyright to your article

Submit your next manuscript at $>$ springeropen.com 